\title{
Tolerancing a solid model with a kinematic formulation
}

\author{
Louis Rivest, Clement Fortin and Claude Morel
}

\begin{abstract}
The $3 \mathrm{D}$ analysis of tolerances is one of the critical functional requirements of solid-modelling systems which is most lacking at the present time. 3D tolerance analysis is most difficult to carry out by hand when it involves geometrical tolerances. A computerized $3 \mathrm{D}$ tolerance analysis is therefore needed, and an appropriate tolerancing model must be devised. The paper first acknowledges the kinematic nature of tolerance-chain analysis. A kinematic formulation of full 3D dimensional and geometrical tolerances is then proposed. The scheme represents all tolerances within a kinematic model, which is compatible with existing standards such as ANSI Y14.5M. The semantics of tolerances is respected, as tolerances are automatically interpreted to yield tolerance zones and datum reference frames. A tolerance chain is modelled as a kinematic chain, thus enabling multiple tolerances to be manipulated to solve $3 \mathrm{D}$ tolerance-chain analysis problems. The implementation of the modelling concept on an exact solid modeller is described. An application of the tolerancing model to the visualization of tolerance zones is also shown.
\end{abstract}

Keywords: tolerancing, tolerance modelling, solid modelling, tolerance analysis

Tolerance modelling and analysis are vital functions which are not properly supported by commercial solid-modelling systems, and are absolutely required for complete product modelling, particularly within a concurrent-engineering environment. Product modelling must therefore incorporate product tolerances, which include dimensional $( \pm)$ and geometrical tolerances. 3D tolerance analysis, when carried out by hand, is most difficult, and sometimes impossible to realize, particularly when it involves geometrical tolerances in a spatial configuration. This analysis is absolutely necessary to

Department of Mechanical Engineering, Ecole PJlytechnique, PO Box 6079, Station Centre-Ville, Montreal, Quebec, Canada H3C 3A7 generate a proper process plan, which specifies how a product must be manufactured. Tolerances, particularly those that are geometrical, influence process planning by imposing precision constraints, which influence the order of the manufacturing operations, particularly the choice of the fixturing surfaces used while some other features are being machined.

Several 3D tolerance-modelling projects have been conducted in the past. Tolerancing theories were first proposed by Requicha and others ${ }^{1-3}$, and were in some cases implemented in solid modellers ${ }^{4}$. Then others, such as Kimura et al., and Johnson et al., developed computerized representation schemes ${ }^{5,6}$. Tolerancemodelling strategies have also been studied by Jayaraman and Srinivasan ${ }^{7}$, Wirtz ${ }^{8}$, and others ${ }^{9-11}$. A few researchers, such as Turner ${ }^{9,10}$ and Martino ${ }^{11}$, also addressed the tolerance-analysis problem of checking whether a set of tolerances respects a design constraint. Other authors, such as Roy and Liu ${ }^{12}$, Shah and Miller ${ }^{13}$, Bernstein and Preiss ${ }^{14}$, Fleming ${ }^{15}$, Clement et al. ${ }^{16}$ and Desrochers ${ }^{17}$, developed methods to represent the relationships between the toleranced features of a part or an assembly.

Few of these models complied fully with actual tolerancing practices on the basis of national or international standards. Even if some authors insisted on standards limitations ${ }^{8,18}$, many others claimed that they were clear and precise communication languages ${ }^{19-21}$. For use in commercial solid-modelling systems, we consider that it is important to support industrial tolerancing practice. Since geometric dimensioning and tolerancing were developed on the basis of long-standing experience, and they have become a universal engineering language ${ }^{20}$, we conclude that a successful tolerancing model must support the standards. A recent paper by Guilford and Turner ${ }^{22}$ proposed a representation that is in line with the ANSI standard.

However, while some publications recognized the importance of capturing the relationship between toleranced features, none of them adequately answered 
the fundamental tolerance-analysis problem formulated by Clement et al. ${ }^{16}$ :

... given an uncertainty zone defined in a reference frame A, how do we compute the value of this uncertainty zone in a new reference frame $\mathrm{B}$, knowing the uncertainty zone associated to the surfaces composing the reference frame?

A tolerance-modelling scheme that supports this kind of tolerance analysis must explicitly capture the 3D topological links induced by tolerances, and allow them to be manipulated. In this paper, we present such a 3D tolerance-modelling scheme, based on a kinematic formulation, which captures the meaning of tolerances, expressed in terms of features constrained into tolerance zones and related to datum features. It complies with the ANSI Y14.5M 1982 tolerancing standard ${ }^{23}$, and it solves 3D tolerance-analysis problems. Although this paper emphasizes the description of the kinematic model rather than its application to tolerance analysis, the kinematic character of the analysis is first presented.

\section{Why use a kinematic formulation?}

In this paper, the tolerancing problem stated above is considered from a manufacturing point of view. However, the similarity between tolerance-analysis problems in design and in process planning ensures the generality of the discussion. The study of tolerance analysis considers the relation established between an 'end feature' and some datum features through a tolerance chain, as defined by its product definition. The analysis aims to replace the tolerance chain with a new single tolerance, a manufacturing tolerance, that directly relates the end feature to the datum features chosen as fixturing surfaces for manufacturing. This new tolerance influences the selection of the machine tool used, and the sequence of operations in the process plan.

The kinematic character of the analysis is best illustrated by an example. Figure 1 shows a simple part which includes some geometrical tolerances. The position of hole 1 is toleranced with respect to the datum features $\mathrm{A}, \mathrm{B}$ and $\mathrm{C}$, while the position of hole 2 is toleranced relative to the axis of hole 1 . Since it makes sense to position the workpiece on features A, B and C (defining the reference frame $X, Y, Z$ ) while manufacturing both holes, the tolerance chain relating hole 2 to $\mathrm{A}, \mathrm{B}$ and $\mathrm{C}$ through hole 1 has to be replaced by a single 'equivalent' tolerance.

The methodology used to obtain the available tolerance of hole 2 relative to $\mathrm{A}, \mathrm{B}$ and $\mathrm{C}$ can be represented by the displacement calculation within a kinematic chain. To properly explain the concept, Figure $l$ is compared with its corresponding kinematic representation shown in Figure 2. The axis of hole 1 is constrained in a cylindrical tolerance zone (zone A) positioned relative to datum planes $\mathrm{A}, \mathrm{B}$ and $\mathrm{C}$. The axis

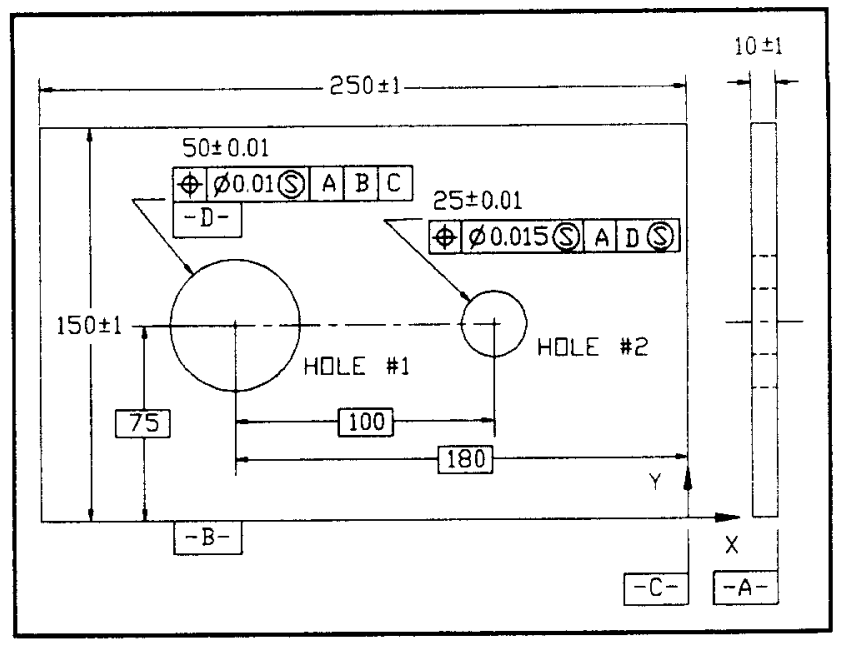

Figure 1 Simple part with geometric tolerances

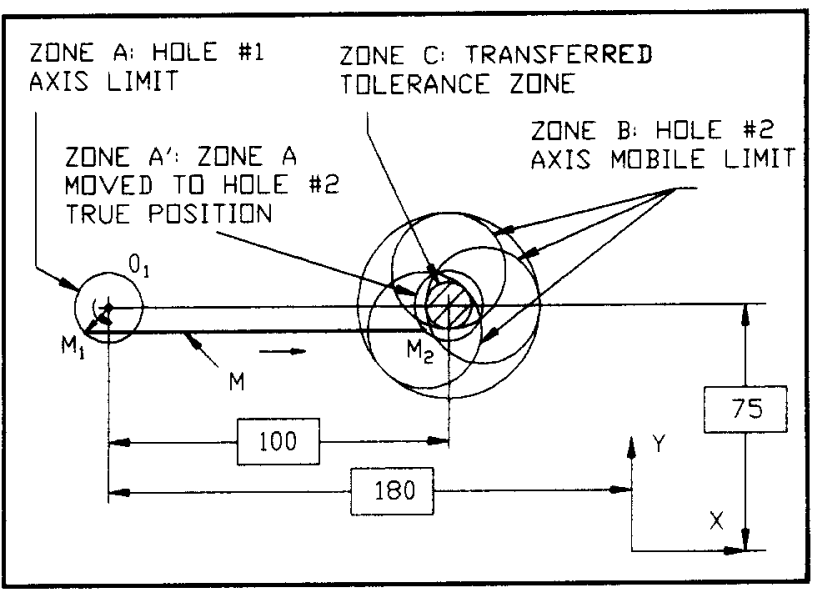

Figure 2 Relating tolerance zones with kinematic link

of hole 2 is constrained in a cylindrical tolerance zone (zone B) positioned relative to a datum system constructed over plane A and the axis of hole 1. For this simple example, in the worst case, the tolerance zone can be represented by allowing vector $\mathbf{O}_{1} \mathbf{M}_{1}$ to rotate within the limit of zone A. As the axis of hole 1 is allowed to move within zone $A$, the position of the axis of cylindrical zone $\mathrm{B}$ may be found anywhere in the zone $\mathrm{A}^{\prime}$ shown in Figure 2, where the rigid link between zones A and B is represented by the thick line $M$. The objective of the calculation is to determine a new tolerance zone relative to datum planes $\mathrm{A}, \mathrm{B}$ and $\mathrm{C}$ within which the axis of hole 2 must lie for the geometrical tolerance of the position applied to hole 2 to be indirectly respected. Obviously, the new zone which meets this requirement is the cylindrical space common to all possible zones B, which is represented by zone $\mathrm{C}$, which is shown crosshatched in Figure 2. This zone is obtained by sweeping point $M_{1}$ of link $\mathrm{M}$ in zone A.

From the kinematic character of the link imposed by a tolerance between its datum and its toleranced feature 
(holes, in this case), a new tolerance-modelling scheme based on a kinematic formulation is proposed. This modelling scheme enables tolerance-analysis problems such as the one described above to be solved, taking into account the effect of modifiers and datum precedence. However, solving such a tolerance-chain analysis problem for a general case raises many issues, which require further research work.

\section{General requirements for tolerance modelling}

The kinematic formulation complies with the following functional requirements identified by Shah and Miller ${ }^{13}$ for a tolerance modeller. It must

- support all the information needed to define all the tolerance classes,

- be extensible,

- support datum reference frames and precedence,

- network tolerances with geometric entities,

- provide validity checking of geometric entities,

- support material modifiers,

- provide automatic checks on the legality of tolerances,

- apply default tolerances to untoleranced entities,

- provide graphic feedback.

It is implied in this list that, for a full 3D tolerance-representation scheme to be capable of tolerance analysis, it must encompass the meaning of tolerances specified by the designer. As shown, the proposed kinematic formulation meets all of the above requirements including this implicit one. It allows the capture of all the tolerances allowed by current standards such as ANSI Y14.5 1982, including dimensional as well as geometrical tolerances. Complex surfaces, controlled by profile tolerances, can also be dealt with by the kinematic formulation. Their tolerance zones are usually defined by a plane in space and pairs of points, lines or arcs which can easily be positioned and oriented with the $3 \mathrm{D}$ kinematic modeller.

The model encompasses all the information relative to a tolerance definition, including the type of tolerance applied, the constrained feature, datum features, and modifiers. Further, it includes the meaning of a tolerance in an explicit way following its capture by an automatic tolerance-interpretation procedure.

This procedure evaluates the global effect on the feature constrained of all the information elements comprised in a tolerance, including

- the description of the toleranced feature,

- the datum coordinate system,

- the effects of modifiers on the datum system,

- the shape and size of the tolerance zone, including virtual surface calculations if relevant,

- the position and orientation of the tolerance zone in the datum system.
This model can also be easily converted to a matrix form, which can then be used to display a tolerance zone on a graphics screen.

\section{TOLERANCE MODELLNG AND SOLID MODELLING}

\section{Technotopological model}

Product-model information is often classified into three categories: the geometric model, the topological model, and the technological model. The geometric model describes the geometry of a part, that is, equations of surfaces, curves and associated coordinates ${ }^{25}$.

When the geometry of a part is modelled with a solid modeller, its definition includes the topological structure of that part. The topological model describes the relations between the part elements and features. The topological knowledge of a part allows one to know to which face a curve belongs, or to check the adjacency of two faces. Topology and geometry are fundamentally related in a product solid model.

The technological model comprises surface finishes and tolerances. Surface finishes are uniquely related to single surfaces, and do not represent a modelling problem. Geometrical tolerances impose constraints on features, located between some theoretical limits, which often are themselves positioned relative to other features of the part. For example, a geometrical tolerance of parallelism may impose a constraint such that a planar face $A$ be found between theoretical planes (the tolerance zone) parallel to a datum planar face B. Such a tolerance constrains the attitude of an element relative to another one, which may be viewed at first as geometrical or topological information. A part geometry implicitly includes equations of surfaces; therefore, this implies that a nominal relationship can be deduced between these surfaces. Besides, topology is often viewed as the knowledge of connectivity between elements of a part. None of these definitions supports the knowledge of the relationship specified by a tolerance between elements. We then propose to enlarge this simplistic definition of topology to include the relationship imposed by tolerances between elements.

This close relationship between the technological and topological models is underlined by the qualification of the proposed tolerancing scheme as a technotopological model. It complements the solid model over which it is built. The concept of technologically and topologically related surfaces (TTRSs) proposed by Clement et al. also recognizes that technological and topological information are closely associated ${ }^{16}$.

\section{Solid-model boundary representation and tolerance modelling}

A technotopological model must rest on an appropriate and exact solid-geometric-model representation. The 


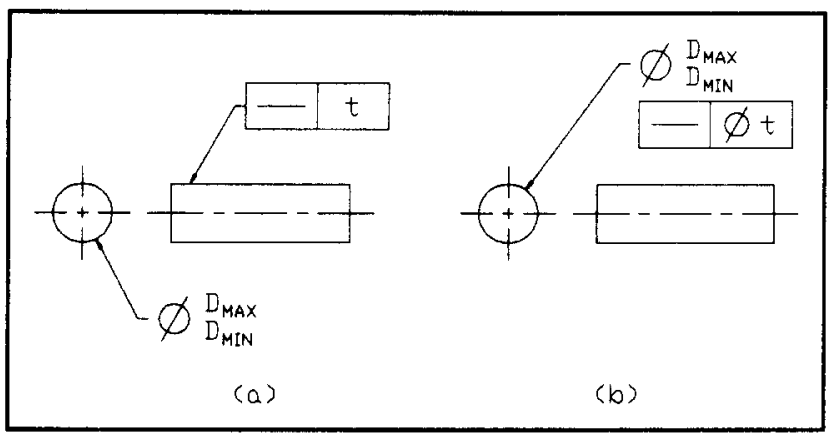

Figure 3 Case in which position of tolerance determines its meaning [Adapted from Reference 24.]

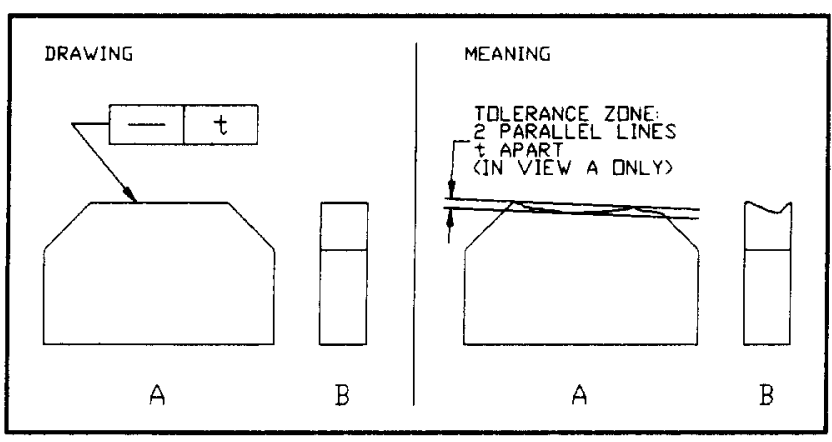

Figure 4 Case in which meaning of tolerance relates to view in which it appears

[Adapted From Reference 24.]

boundary representation of a solid is the most appropriate, because tolerances relate to product faces. However, as shown below, a face identifier alone may fail to capture the meaning of some tolerances.

A tolerance either applies to the symmetry element of a face (the axis of a cylindrical feature of size, or the centre plane of a planar feature of size), or to a face itself. For example, a straightness tolerance may be applied to a cylindrical-surface generator (see Figure $3 a$ ) or to its axis (see Figure $3 b$ ). The position of the feature control frame indicates whether the tolerance, in this case, is to be applied to the face or to its axis. Consequently, relating tolerances to a part surface alone does not always suffice, since the nature of the constrained element may be needed in some cases. This supplementary information is included in the proposed technotopological model.

In some cases, it is also important to note that the meaning of some geometrical tolerances relates to the view in which they appear. For example, the straightness tolerance of Figure 4 requires that each of the top planar-face line segments which are parallel to the projection plane in view A be found between a pair of parallel straight lines. This tolerance does not impose any condition on view $B$. Thus the meaning of some tolerance relates to a $2 \mathrm{D}$ projection of the geometry of a part, while a solid geometric model of a part is $3 \mathrm{D}$, and is independent of the visualization.

For Srinivasan ${ }^{18}$, the problem is seen as a syntactic limitation of standards. We consider it rather as a syntactic correspondence problem between a technotopological constraint partly defined by a $2 \mathrm{D}$ projection of a part, and a 3D solid model. It can be overcome by encapsulating the projection vector for view A of Figure 4 (normal to the paper), thus rendering it a component of the tolerance definition. The orientation of the constrained line segment on the top plane is then considered normal to the projection vector.

\section{KINEMATIC FORMULATION}

\section{General description of kinematic formulation}

The kinematic model defines the element of a part that is constrained by a tolerance as the object of that tolerance. It can be a group of faces, a face, a part of a face, or the symmetry element of a face (the axis of a cylindrical surface etc.). For the tolerance description to be complete, the object constrained, as well as the tolerance zone, must be characterized. As stated above, a tolerance zone is defined by five pieces of information: its shape (cylindrical, planar, spherical etc.), its size, its datum system, its position, and finally its orientation relative to the datum system.

The description of tolerance zones is encapsulated within the kinematic structure, which is shown in Figure 5. Here, each block and cylinder represents one degree of freedom or a parameter, in translation and rotation, respectively. Rotation-parameter identifiers start with an $R$, and translation-parameter identifiers start with a $T$. The space limited by a given tolerance zone corresponds to the region of space that is accessible to the point $O_{\mathrm{f}}$ of the structure when many of its parameters are set to fixed values, while the others are free to sweep within their given intervals. Therefore, modelling a tolerance

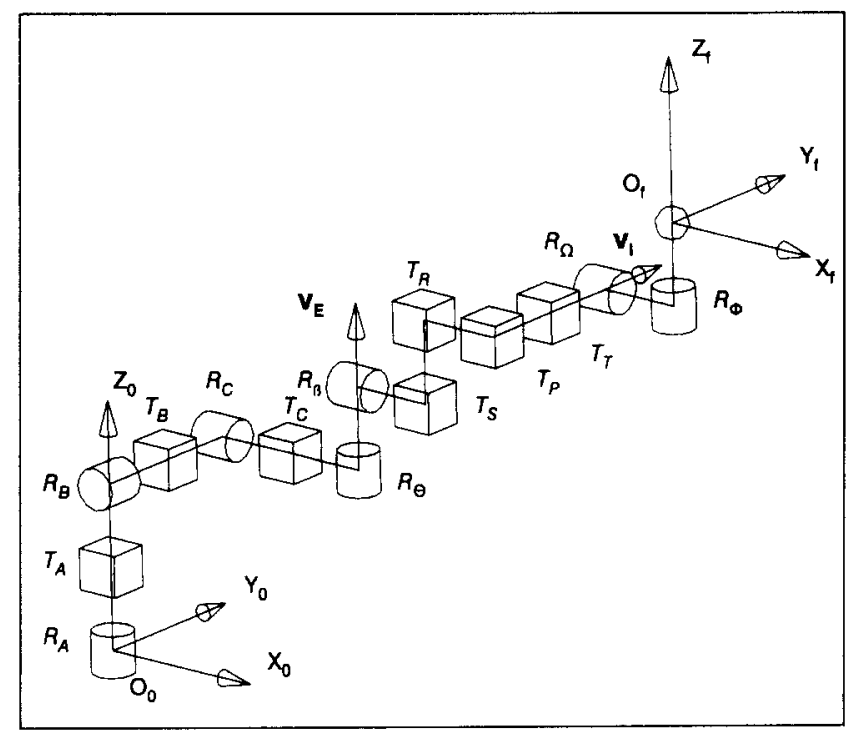

Figure 5 Kinematic structure 
zone is equivalent to finding an appropriate set of values to initialize 14 parameters of the structure.

The proposed structure can be used to model all the tolerance zones allowed by current standards, as discussed by Rivest, Fortin and Desrochers ${ }^{26}$, and it considers the effect of modifiers used with geometrical tolerances. For example, a geometrical tolerance of position applied to a hole at the maximum-material condition (MMC) specifies a solid cylindrical tolerance zone, meaning one where a male gauge (inside) is used to control the tolerance. The tolerance zone determined for such an example precisely defines the size and type of the gauge used for tolerance control. In contrast, a hollow tolerance zone is one which requires a female (outside) gauge. This distinction between solid and hollow tolerance zones is one component of the method used to direct the interpretation of tolerances. However, if the tolerance is applied regardless of feature size (RFS), it specifies a hollow cylindrical tolerance zone within which the axis of the hole must be found. As the kinematic formulation rests on tolerance zones, dimensional $(+/-)$ tolerances can also be modelled within that scheme if they are interpreted as tolerance zones; this topic is discussed in more detail in a following section.

As the tolerance zone specified by a tolerance is a function of the modifiers used, so is the object constrained. Consequently, the object of a tolerance is not completely characterized by its face identifier, as the nature of the toleranced feature must also be specified. The nature of a feature distinguishes the face itself from its symmetry element. In the last example, the object constrained at the MMC is the surface itself, while, in another case, the object constrained RFS is the symmetry element of the designated cylinder: its axis. For a planar feature of size, such as a slot, the symmetry element is its centre plane, while the feature surface is the pair of faces themselves. When a simple planar face is designated as an object, the toleranced element is the planar face itself. The effect of a tolerance application modifier is thus modelled not only by the type of the tolerance zone defined, but also by the nature of the toleranced feature.

\section{Kinematic-structure parameters}

The modelling of a given tolerance zone is carried out by setting the parameters of the kinematic structure to appropriate values to allow the origin of the end coordinate system $\left(O_{\mathrm{f}}\right)$ to move anywhere in the space of the tolerance zone. By merely changing the magnitude of the parameters, the structure described in Figure 5 can be used to represent the variety of tolerance zones allowed by standards and listed by Rivest, Fortin and Desrochers ${ }^{26}$. It is important to describe each parameter of the model to understand the general applicability of the kinematic structure to tolerance modelling and analysis.

The analytical tolerance zones admitted by the ANSI standard are classified into two categories: cylindrical and planar ${ }^{26}$. Similarly, the orientation and position of tolerance zones are characterized by their symmetry elements: the axis for a cylindrical tolerance zone, and the median plane for a planar tolerance zone. The orientation of the axis of a cylindrical tolerance zone is given by the axis vector, while the position of such a zone is given by a vector to a point on the axis. The orientation of a median plane is given by its normal vector, while its position is given by a vector to a point on the plane. The vector characterizing the orientation and position of a tolerance zone relative to its datum system is called an external vector $\mathrm{v}_{\mathrm{E}}$. Parameters $R_{\mathrm{A}}, T_{\mathrm{A}}, R_{\mathrm{B}}, T_{\mathrm{B}}, R_{\mathrm{C}}$ and $T_{\mathrm{C}}$ control the true position and orientation of the external vector of a tolerance zone. On the kinematic structure, this corresponds to the axis of parameter $R_{\Theta}$, shown as $\mathbf{v}_{\mathrm{E}}$ in Figure 5.

An internal vector $\mathbf{v}_{\mathrm{I}}$, along which the size of a tolerance zone is measured, is also defined. The relationship between the internal and external vectors characterizes the shape of a tolerance zone. The parameters $R_{\Theta}$ and $R_{\beta}$, which are two orthogonal rotations, and parameters $T_{\mathrm{S}}$ and $T_{\mathrm{R}}$, two translations, control this relationship within the structure. A cylindrical zone is modelled by setting $R_{\Theta}$ within an interval $[0,2 \pi]$ and $R_{\beta}$ to zero, while $T_{\mathrm{S}}$ can represent its length, and $T_{\mathrm{R}}$ is set to zero. This places the vector $\mathbf{v}_{\mathbf{l}}$, which supports the parameter $T_{\mathrm{T}}$, perpendicular to vector $\mathbf{v}_{\mathrm{E}}$, rendering it a turning vector. A planar zone is modelled by setting $R_{\Theta}$ to zero and $R_{\beta}$ to $\pi / 2$ for the vector $v_{1}$ to be parallel to $v_{\mathrm{E}}$, while $T_{\mathrm{S}}$ and $T_{\mathrm{R}}$ can be used to represent the width of the zone.

The parameter $T_{\mathrm{P}}$ characterizes zones as solid or hollow. A solid zone defines a portion of space from which the constrained object must be excluded, as in the case of a hole excluded from a solid cylindrical zone. In such a case, $T_{\mathrm{P}}$ is set to the radius of the virtual condition of the hole. The parameter $T_{\mathrm{P}}$ is, on the other hand, set to zero in the modelling of hollow zones. The parameter $T_{\mathrm{T}}$ determines the size of a zone, being, for example, the distance between the limits of a planar zone, or the radius of a hollow cylindrical zone.

The last two rotation parameters $R_{\Omega}, R_{\Phi}$ control the orientation of the end coordinate system $\left(O_{\mathrm{f}}, X_{\mathrm{f}}, Y_{\mathrm{f}}, Z_{\mathrm{f}}\right)$ relative to the external vector, and they are required for analysis.

\section{Placing kinematic structure on solid-model features}

Initializing the kinematic model requires the placement of its basic coordinate system $\left(O_{0}, X_{0}, Y_{0}, Z_{0}\right)$ onto the solid geometric model of the part. To do this, a datum coordinate system is first constructed from the set of datum features of a given tolerance. The basic coordinate system of the kinematic model is then made coincident with it. A datum is defined here as a theoretically exact point, axis or plane derived from the geometric model of a specified datum feature. Further, a datum feature is a feature of a part that is used to establish a datum. The 
Tolerancing a solid model with a kinematic formulation: L Rivest et al.

Table 1 Datum coordinate system construction rules summarized for one case of datum association

\begin{tabular}{|c|c|c|c|c|c|}
\hline $\begin{array}{l}\text { Symbolic } \\
\text { representation }\end{array}$ & Conditions & $\begin{array}{l}\text { DCS } \\
\text { origin }\end{array}$ & $\begin{array}{l}\text { DCS } \\
\text { orientation }\end{array}$ & $\begin{array}{l}\text { Basic } \\
\text { dimensions }\end{array}$ & \\
\hline$A\left|\begin{array}{ll} & \\
z_{0} & \end{array}\right|$ & $\begin{array}{l}\mathbf{v}_{\mathrm{A}} / / \mathbf{n}_{\mathrm{B}} \\
\mathbf{v}_{\mathrm{A}} / / \mathbf{v}_{\mathrm{C}}\end{array}$ & $\begin{array}{l}\text { On axis } A \\
\text { On plane B }\end{array}$ & $\begin{array}{l}Z_{0} \text { orientation }=\mathbf{v}_{\mathbf{A}} \text { orientation } \\
Z_{0} \text { direction }=\mathbf{n}_{\mathbf{B}} \text { direction } \\
Y_{\mathrm{o}} \text { orientation } \equiv \mathbf{v}_{\mathrm{c}} \\
Y_{\mathrm{o}} \text { direction } \equiv \mathbf{v}_{\mathbf{C}}\end{array}$ & $\begin{array}{l}\text { Related } \\
\text { to datum } \\
\text { A } \\
\text { B } \\
\text { C }\end{array}$ & $\begin{array}{l}\text { Measured } \\
\text { along axis } \\
Y_{0} \\
Z_{0} \\
X_{0}\end{array}$ \\
\hline
\end{tabular}

[A: primary datum, B: secondary datum, C: tertiary datum, DCS: datum coordinate system, $\mathbf{v}_{\mathrm{A}}$ : primary-datum axis vector, $\mathbf{n}_{\mathrm{B}}:$ secondary-datum plane normal vector, $\mathbf{v}_{\mathrm{C}}$ : tertiary-datum axis vector, $X_{0}, Y_{0}, Z_{0}$ : datum coordinate system axis, $=$ : axis vector takes value of designated vector, $\equiv$ : axis vector goes from origin to designated vector, orientation: characterizes equation of line supporting axis, direction: characterizes positive side of line and, accordingly, of axis.]

datum associated with a cylindrical feature is thus its theoretical axis, while the datum associated with a planar feature of size is its theoretical centre plane, and the datum associated with a planar face is the theoretical plane. A datum is represented by its characteristic vector, i.e. the vector normal to a theoretical plane or centre plane, and the axis vector in the case of a theoretical axis.

A datum coordinate system is constructed on a geometric model by combining, in a systematic manner, the datum vectors obtained from the datum features specified by a tolerance. Several practical cases demonstrating the association of datum features have been studied, and construction rules have been chosen for each one. Table 1 summarizes such rules for the case in which a cylindrical feature of size, a planar face and a second cylindrical feature of size are used as primary, secondary and tertiary datum features, respectively, and their characteristic vectors are parallel. An example using this association of datum features is detailed in the next section.

Once the datum coordinate system has been defined, the modifiers eventually applied to datum features of size must be captured within the kinematic formulation concept. A modifier applied to a datum feature of size specifies the way in which its datum may deviate from its true position. When an RFS modifier is applied to a feature of size, the datum is allowed to deviate from its true position by an amount specified by another tolerance controlling this feature of size. However, if an MMC modifier is applied, the datum is allowed to deviate from its true position by an amount that is limited by the feature-of-size virtual condition, and a datum shift is then said to be available. Consequently, the effect of modifiers on datum features of size is modelled by determining whether the allowed deviation of a datum from its true position is limited by either the feature-of-size symmetry element, or the surface.

\section{Modelling example}

In this section, the kinematic formulation used to model the geometric tolerance of position applied to the slot on

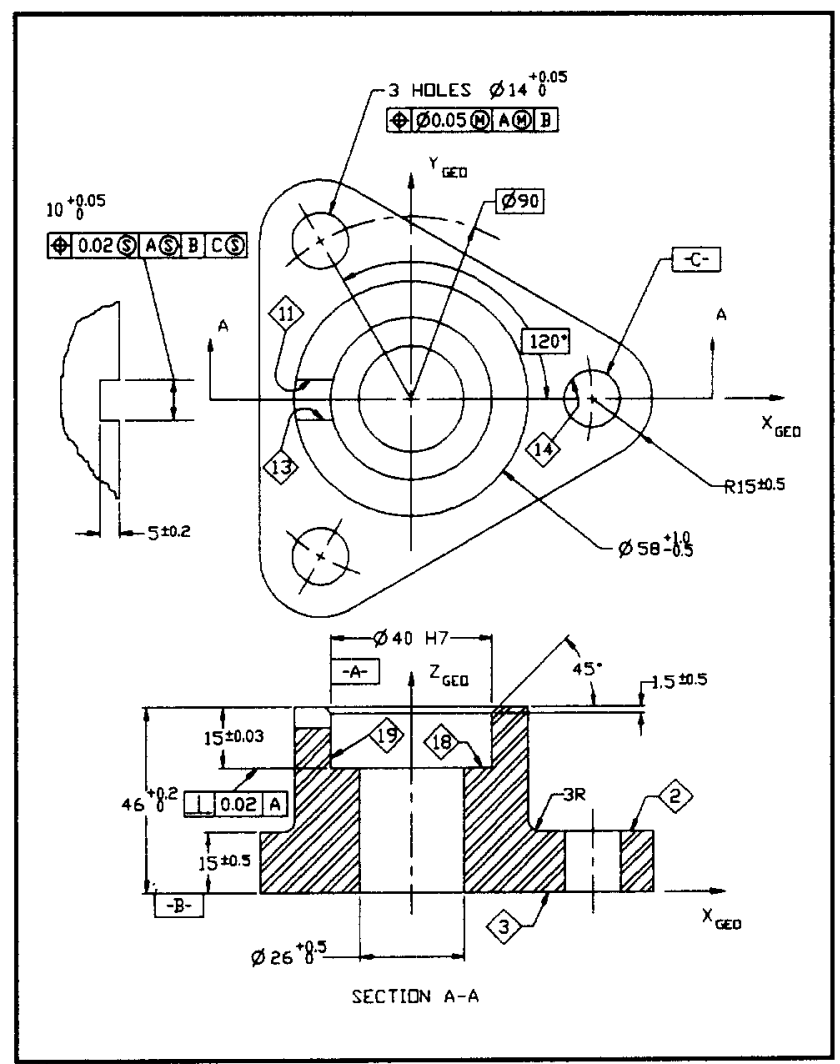

Figure 6 Sample part with tolerances to be modelled

the part in Figure 6 is examined. The type of tolerance zone specified and the nature of the toleranced feature are first identified. As the tolerance is applied RFS, it constrains the slot centre plane (the object) within a planar tolerance zone. The zone is placed in a datum coordinate system (see Figure 7) constructed over a cylindrical feature-of-size datum, a planar-feature datum and a second cylindrical feature-of-size datum, their characteristic vectors being parallel. According to Table 1 , the origin of this coordinate system is placed at the intersection of the primary datum and secondary datum (axis A and plane B). Its orientation is also determined according to Table 1 . 


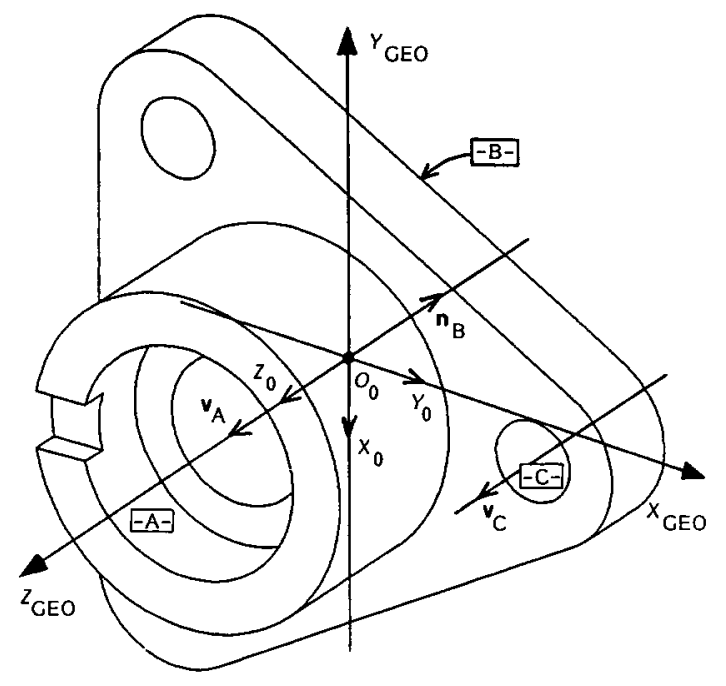

Figure 7 Datum coordinate system constructed over datum features $A, B$ and $C$

In Table 1, there is a distinction between the orientation and the direction of a vector: the orientation characterizes the equation of a line, while its direction characterizes the positive side of this line. In the case under discussion, the orientation of the $Z_{0}$ axis is determined by the primary datum, which is represented by its axis vector $\mathbf{v}_{\mathrm{A}}$. However, as the axis of a cylinder does not possess a positive side, its vector cannot specify the direction of the $Z_{0}$ axis. The secondary datum, as a plane, is characterized by its normal vector $\mathbf{n}_{\mathrm{B}}$, whose direction indicates the space side (exterior of the part). Where both vectors are parallel, the positive side of the $Z_{0}$ axis is determined by the second vector; thus, in this case, its direction is determined by $\mathbf{n}_{\mathbf{B}}$. However, by convention, the direction of the $Z_{0}$ axis indicates the part side so that its direction, as shown in Figure 7 , is the inverse direction of $\mathbf{n}_{\mathbf{B}}$. In this case of association of datum features, the $Y_{0}$ axis is supported by a vector going from the point of origin of the datum coordinate system to the axis of the second cylindrical feature of size (tertiary datum). The $X_{0}$ axis is easily obtained from the first two.

When the tolerance zone and the object are known, and the datum coordinate system is constructed, the tolerance zone is modelled by setting the kinematicstructure parameter values. The parameters $R_{\mathrm{A}}, T_{\mathrm{A}}, R_{\mathrm{B}}$, $T_{\mathrm{B}}, R_{\mathrm{C}}$ and $T_{\mathrm{C}}$ must be initialized so that the vector $\mathbf{v}_{\mathrm{E}}$ of the kinematic structure, as shown in Figure 5, can be positioned and oriented relative to the basic coordinate system $\left(O_{0}, X_{0}, Y_{0}, Z_{0}\right)$ in such a way as to represent the position and orientation of the tolerance zone with respect to the datum coordinate system. The external vector of a planar tolerance zone is normal to the zone median plane, which is, itself, parallel to the limit planes of the zone. In this case, the planes are parallel to the faces of the slot. The solid geometric model of the part specifies that the characteristic vector of the slot faces is parallel to the $Y_{\mathrm{GEO}}$ axis of the coordinate system in which the geometric model is defined (see Figure 6). As $Y_{\text {GEO }}$ is parallel to the $X_{0}$ axis of the basic coordinate system (see Figure 7), the kinematic-structure vector $v_{\mathrm{E}}$ must be parallel to $X_{0}$. Thus the parameters $R_{\mathrm{A}}, R_{\mathrm{B}}$ and $R_{\mathrm{C}}$ of the kinematic structure are initialized to the values $-\pi / 2$, 0 and $-\pi / 2$, respectively. Since the side length of this planar tolerance zone (if only the positional tolerance is considered) is undefined, the position of the zone along $Y_{0}$ and $Z_{0}$ does not have any meaning; thus $T_{\mathrm{A}}$ and $T_{\mathrm{C}}$ are arbitrarily set to zero. The position of the planar zone along its normal vector, which in this case is $X_{0}$, must be specified by the tolerance. In this example, the median plane of the zone coincides with the origin of the basic coordinate system; thus the parameter $T_{\mathrm{B}}$ is also set to zero.

The next step aims at modelling the shape of the tolerance zone with the parameters $R_{\Theta}, R_{\beta}, T_{\mathrm{S}}$ and $T_{\mathrm{R}}$. The zone is planar, and therefore the first two parameters take the values 0 and $\pi / 2$, respectively, such that the vector $v_{1}$ is parallel to the vector $\mathbf{v}_{\mathrm{E}}$ (see Figure 5). The parameters $T_{\mathrm{S}}$ and $T_{\mathrm{R}}$ measure the side length of the zone, which is undefined for now, and, like $T_{\mathrm{A}}$ and $T_{\mathrm{C}}$, they are set to zero. The parameter $T_{\mathrm{P}}$ is set to zero for a hollow zone, while the parameter $T_{\mathrm{T}}$ is kept within an interval $[-0.01,0.01]$. In this example, the last two rotation parameters are set to $-\pi / 2$ and zero, respectively, such that the $Z_{\mathrm{f}}$ axis is parallel to vector $\mathbf{v}_{\mathrm{l}}$, which will be useful later.

The last modelling step addresses the modelling of the effect of modifiers applied to feature-of-size datums. This is done by determining whether the freedom between a datum and its true position is limited, either by its symmetry element or by its surface, as explained above. In this example, both feature-of-size datums are used RFS, and thus their freedom relative to their true position is limited by their symmetry elements.

\section{Dimensional-tolerance modelling}

As the kinematic-modelling scheme partially rests on tolerance-zone modelling, and as the tolerance-zone concept is not inherent to dimensional tolerances, one may raise the question about the ability of this scheme to model dimensional tolerances.

Dimensional tolerances are classified into two categories: dimensional tolerances of location, and dimensional tolerances of size. The first type imposes a dimensional relation between two features, such as two parallel planes, while the second type is reserved for specifying the size of a feature of size. The modelling of dimensional tolerances relating two features requires one of the features to be the datum feature, while the other is the toleranced feature.

According to tolerancing standards, dimensional tolerances do not always specify a precedence between the related features. They thus neither bear the concept of datum and object, nor involve the concept of a tolerance zone where the object must lie. Instead, a local 
measure of distance at various points between the related features must be inside acceptable limits.

Modelling this type of tolerance with a kinematic scheme requires the user to choose one of the two features to be a datum and the other to be the object. There is no problem with this methodology, since the datum and object concepts are most often present in the mind of a designer who specifies a dimensional tolerance of location. In Figure 6, for example, the tolerance $15 \pm 0.5$ relating planar faces 2 and 3 (datum feature B) clearly indicates that face 3 must be a datum, and face 2 the object. The dimensioning of a planar feature of size, like the slot in Figure 6, does not yet imply an inherent datum-object relation. There is no obvious precedence between these faces. However, choosing a datum does not raise a problem even in this case, since the dimensional tolerance is subordinate to the geometrical tolerance of position, controlling the slot as a symmetrical feature

The kinematic modelling of dimensional tolerances relating two features involves enclosing the chosen object within a hollow planar tolerance zone. This departs from current standards by adding the requirement for a feature of size to be of perfect form at the least-material condition. According to current standards (ANSI), when such a tolerance is imposed alone on a feature of size, it is required to be of perfect form at the $\mathrm{MMC}$, and to fit, when at the least-material condition, inside the envelope obtained at the MMC. This departure from the standard is assessed as minor and acceptable.

Dimensional tolerances of size controlling a diameter can be considered in a similar manner. Modelling is carried out using the axis of a cylindrical feature of size as a datum, while its surface is constrained within a tolerance zone limited by two cylindrical surfaces sharing the same axis. This corresponds to the converting of dimensional tolerances of size into cylindricity tolerances. As previously stated, the kinematic modelling of such dimensional tolerances of size departs from standards by adding the requirement that the surface be of perfect form at the least-material condition. Again, we evaluate this departure from the standard as minor and acceptable.

\section{KINEMATIC-MODEL IMPLEMENTATION}

A tolerance-modelling prototype was built to validate the kinematic formulation. The tolerance-modelling scheme was added to the solid geometric model of a part. As the tolerance model was particularly intended for process planning, it was assumed that the geometric model was frozen before the tolerance model was added to the part model: the solid geometric model was therefore considered static. Thus, the relationship between the tolerancing model and the solid geometric model was simplified.

The tolerancing scheme used the boundary representation of a PADL-2 solid geometric model ${ }^{27}$. However, the tolerance modeller was developed as an independent module which could be integrated into any solid modeller, such as $\mathrm{ACIS}^{28}$. The boundary representation was stored in a database designed for the tolerancemodelling module. It contained

- an identifier for each face of the solid geometric model

- a description of each face, characterized by

- a code indicating whether it was cylindrical or planar,

- its characteristic orientation vector,

- a position vector,

- for cylindrical faces, a code indicating whether the feature was male or female.

The prototype was used to model tolerances of the part shown in Figure 6 . The functional diagram of the prototype (see Figure 8) shows that tolerances are modelled after completion of the solid geometric model. A user interface was designed for the interactive description of tolerances. Each tolerance is first stored in

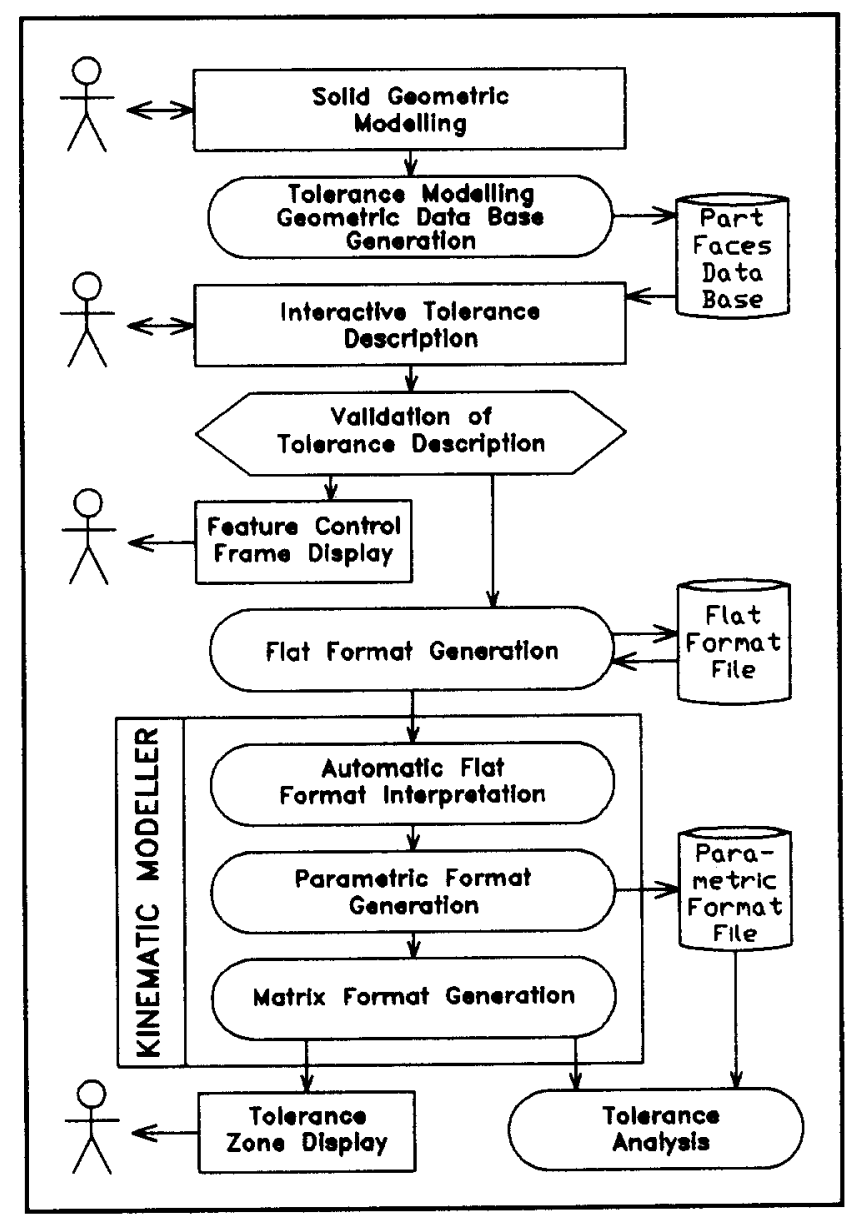

Figure 8 Tolerance-modelling prototype 
a flat format, which is then automatically interpreted to generate an appropriate tolerance description in a parametric format. This format is then transformed into a matrix format, which shows the tolerance zone on the part appearing on a graphics screen.

\section{Interactive tolerance description}

An interactive menu and a graphical interface guide the user in the definition of tolerances. Displaying the part simultaneously along four different user-chosen projections has enhanced the PADL-2 solid-modeller graphics facilities. The user describes a tolerance by first choosing from a menu the type of tolerance to be modelled (for example a geometrical tolerance of position). He/she then selects the object of this tolerance and the datum features with a pointer on the graphics screen. $\mathrm{He} / \mathrm{sh}$ also indicates which modifiers are to be applied, if relevant. Finally, he/she indicates the tolerance value and the basic dimensions associated with datums. While the user describes a tolerance, various syntactic validations are automatically carried out (see Figure 8). For example, the object of a geometric tolerance of position is checked to make sure that it is a feature of size, as required by the ANSI standard. Upon completion of a tolerance description, its feature control frame is automatically constructed, according to the ANSI standard in that prototype, and drawn in a graphics window, allowing the user to check that the description conforms to its intention.

The description of a tolerance given by the user is first stored in a flat format before it is automatically transformed to a kinematic model. The kinematic-model representation resides in parametric and matrix formats. These three formats are outlined below.

\section{Flat formats}

Flat formats have been designed to store the tolerance description obtained from the user directly. One flat format exists for each type of tolerance, and it contains all the information necessary to describe a tolerance completely. Some flat formats used to store the tolerances of the sample part of Figure 6 are shown in Table 2. The TABTOL format, designed for dimensional tolerances of location, includes four information elements: object and datum feature identifiers, the median location dimension, and the tolerance value. In Table 2, the TABTOL format describes the dimensional tolerance of location specifying the slot size as an object face (13) and a datum face (11). The median distance between these faces is $10.025 \mathrm{~mm}$, with a range of $0.050 \mathrm{~mm}$ equally distributed on each side of this distance. The TGPOSIT flat format is used in Table 2 to store the description of the geometric tolerance of position applied to the slot (see Figure 6). The object identifier (501) refers to the complementary format, TGPLNFOS, with which planar features of size are described. The settings of tolerance modifiers are described by entries $S H, T M, M_{1}, M_{2}$ and $M_{3}$ (as detailed in the footnote to Table 2). The tolerance value is indicated by Tolval, which is set here to 0.02 . Entries $D A_{1}, D A_{2}$ and $D A_{3}$ identify datum features. In this case, the primary datum feature is face 19 (datum feature $A$ in Figure 6), as indicated by entry $D A_{1}$. Geometric definitions of datum features are obtained from the database of part faces to construct the datum coordinate system, summarized in Table 1 , for this particular case of datum association. The basic dimensions used to specify the true position of the tolerance zone relative to the datum coordinate system are given by the BSCSIZ entries. For this particular case, Table 1 also specifies how each basic dimension is to be measured in the datum coordinate system.

Table 2 Some flat formats initialized for modelling sample-part tolerances

\begin{tabular}{|c|c|c|c|c|c|c|c|c|c|c|c|c|c|c|}
\hline Format & $O B J$ & $D A T$ & $M E D I A N$ & $I T$ & & & & & & & & & & \\
\hline \multirow[t]{2}{*}{$\begin{array}{l}\text { TABTOL: dimensional } \\
\text { tolerances of location }\end{array}$} & 13 & 11 & 10.02500 & 0.05000 & & & & & & & & & & \\
\hline & $O B J$ & $S H$ & TOLVAL & $T M$ & $D A_{1}$ & $M_{1}$ & $B S C S I Z_{1}$ & $D A_{2}$ & $M_{2}$ & $B S C S I Z_{2}$ & $D A_{3}$ & $M_{3}$ & $\mathrm{BSCSIZ}_{3}$ & $N D$ \\
\hline \multirow[t]{2}{*}{$\begin{array}{l}\text { TGPOSIT: geometrical } \\
\text { tolerances of position }\end{array}$} & 501 & 0 & 0.02000 & 1 & 19 & 1 & 0.00000 & 3 & 0 & 0.00000 & 14 & 1 & 0.00000 & 3 \\
\hline & FOS & $F C_{1}$ & $F C_{2}$ & $G N D$ & & & & & & & & & & \\
\hline $\begin{array}{l}\text { TGPLNFOS: planar } \\
\text { features of size IDs }\end{array}$ & 501 & 13 & 11 & 0 & & & & & & & & & & \\
\hline \multicolumn{15}{|c|}{ 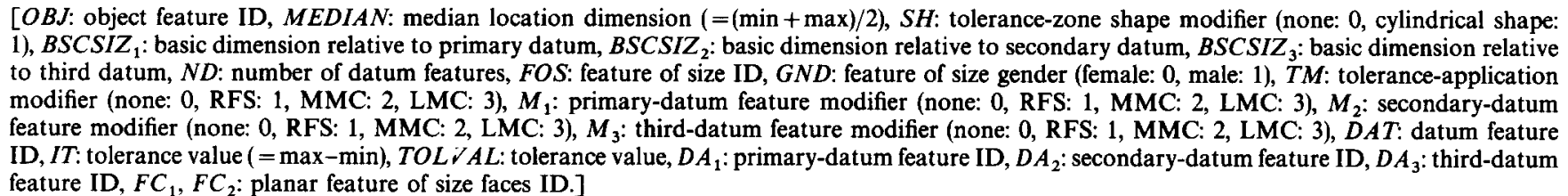 } \\
\hline
\end{tabular}


Tolerancing a solid model with a kinematic formulation: L Rivest et al.

Table 3 Parametric format initialized for modelling geometric tolerance of position applied to slot of sample part

\begin{tabular}{|c|c|c|c|c|c|c|}
\hline$O B J F I D$ & $O B J S R F$ & $O B J S Y M$ & $V Z O D F I D$ & VZOSFID & VYODFID & VYOSFID \\
\hline 501 & $\mathrm{~F}$ & $\mathrm{~T}$ & 19 & 3 & -14 & -14 \\
\hline$O R G O N A X$ & ORGONPL & $D A T S R F$ & DATSYM & $R A$ & $T A$ & $R B$ \\
\hline 19 & 0 & $\mathrm{~F}$ & $\mathrm{~T}$ & -90.00000 & 0.00000 & 0.00000 \\
\hline 0 & 3 & $\mathrm{~T}$ & F & -90.00000 & 0.00000 & 0.00000 \\
\hline 0 & 0 & $F$ & $\mathrm{~T}$ & & & \\
\hline$T B$ & $R C$ & $T C$ & RTHETA & RBETA & $T S$ & $T R$ \\
\hline 0.00000 & -90.00000 & 0.00000 & 0.00000 & 90.00000 & 0.00000 & 0.00000 \\
\hline 0.00000 & -90.00000 & 0.00000 & 0.00000 & 90.00000 & 0.00000 & 0.00000 \\
\hline$T P$ & $T T$ & $R O M E G A$ & $R P H I$ & & & \\
\hline 0.00000 & -0.01000 & -90.00000 & 0.00000 & & & \\
\hline 0.00000 & 0.01000 & -90.00000 & 0.00000 & & & \\
\hline
\end{tabular}

[OBJFID: object feature ID, OBJSRF: TRUE if object is surface of toleranced feature, OBJSYM: TRUE if object is symmetry element of toleranced feature, VZODFID: ID of datum feature imposing $Z_{0}$-axis orientation, VZOSFID: ID of datum feature imposing $Z_{0}$-axis direction, $V Y O D F I D$ : ID of datum feature imposing $Y_{0}$-axis orientation, $V Y O S F I D$ : ID of datum feature imposing $Y_{0}$-axis direction, $O R G O N A X$ : IDs of cylindrical datum features on which origin must lie, ORGONPL: IDs of planar datum features on which origin must lie, DATSRF: true for datums whose deviation is limited by their surfaces, DATSYM: true for datums whose deviation is limited by their symmetry element. Other entries show values of the kinematic structure parameters.]

\section{Parametric format}

As shown in Figure 8, flat-format descriptions of tolerances are read by the kinematic modeller, which automatically interprets them to generate a kinematic model for each tolerance. The parametric format is used to store this interpreted description of a tolerance. An interpreted tolerance description includes the object identifier and its nature, the tolerance-zone description, and the datum coordinate system constructed over the datum features, taking into account the influence of modifiers.

The parametric format generated by the geometric tolerance of position applied to the slot of the sample part (see Figure 6) is shown in Table 3. The first block captures the object identifier and its nature. Here, the number 501 identifies the planar feature of size corresponding to the flat format. The logical entries $O B J S R F$ and OBJSYM, set to FALSE and TRUE, respectively, indicate that the object is the symmetry element of the feature of size. This is because the tolerance is applied RFS, as indicated by the flat format, as mentioned above.

The next two blocks indicate how the datum coordinate system is to be constructed, according to the rules of Table 1, for this particular case of association of datum features. As a reminder, the entry VZODFID indicates that the orientation of the $Z_{0}$ axis of the datum coordinate is imposed by the datum associated with a datum feature whose face number is 19 .

For this example, the third block indicates that the origin of the datum coordinate system is to be found at the intersection of the datum associated with datum feature 19 and the other datum associated with datum feature 3. The fourth block indicates, for each datum feature, whether its maximum deviation from its true position is constrained by its symmetry element or by its virtual condition, as discussed above. Logical entries are used as mentioned above for the object. The other blocks show the values allocated to the parameters of the kinematic structure to model the tolerance zone deduced from the flat-format interpretation.

To summarize, the parametric format is the main representation of the kinematic model, and it is initialized with values that are automatically deduced from the information stored in the flat format. For a given tolerance, it captures the object identity and nature, the tolerance-zone shape and size, the datum coordinate system, and the tolerance-zone position and orientation in that datum system. The kinematic structure proposed is sufficient to model every tolerance and every tolerance zone supported by standards. The kinematic model accounts for the effect of tolerance modifiers, as its initialization process calculates the virtual condition of the object and datum surfaces, where needed.

\section{Matrix format}

The matrix format is the second internal representation of the kinematic model, and it is automatically obtained from the parametric format (see Figure 8). The format is used to display zones in graphic windows, as requested by the user, and also for tolerance analysis.

The format is obtained by converting each parameter of the kinematic structure (see Figure 5) to a homogeneous transformation matrix. These matrices are concatenated to obtain a global transformation matrix which calculates the orientation and position of the final coordinate system relative to the datum coordinate system. A 


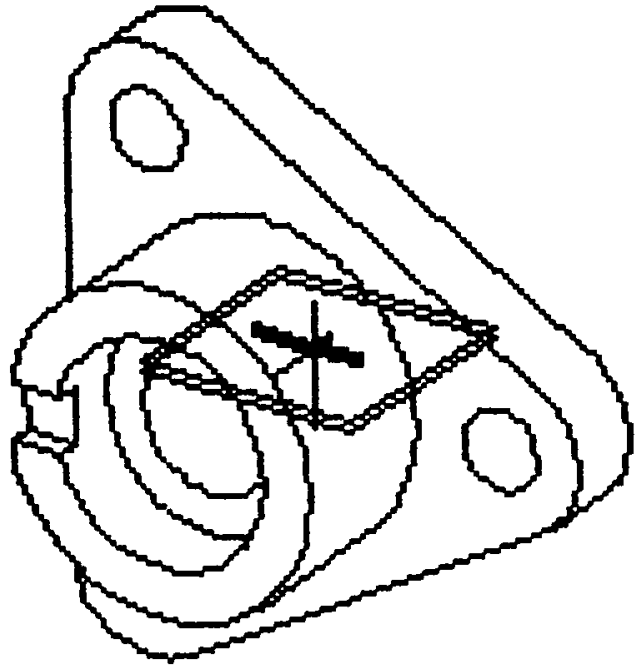

Figure 9 Tolerance zone automatically deduced and displayed by prototype

tolerance zone is displayed by calculating a global transformation matrix for a number of cases representing critical combinations of parameter values describing the kinematic structure. This procedure effectively sweeps the kinematic chain in space, and it can be used to display any shape and size of tolerance zones allowed by the ANSI standard.

Figure 9 shows the planar tolerance zone constraining the centre plane of the sample part slot, as calculated from the parametric format of Table 3 and displayed to the user. For a planar zone, two global matrices are calculated, one for each extreme value of parameter $T_{\mathrm{T}}$, and a symbolic plane is drawn for both cases. A simple scaling procedure is used to display the tolerance zone appropriately.

The display of tolerance zones on request allows the user to check the correspondence between the intended meaning of a tolerance and the constraint actually specified. It may also help a novice user to visualize the meaning of a tolerance. The matrix format is also of interest for tolerance analysis, as tolerances can be linked to one another by simple matrix multiplications.

\section{CONCLUSIONS}

As shown, the kinematic formulation is able to represent dimensional and geometrical tolerances fully in $3 \mathrm{D}$. The methodology, which represents all tolerances with a kinematic chain within a technotopological model, is compatible with all cases of existing standards such as ANSI Y14.5M, and it can be applied within a solid-modelling framework. The semantics of tolerances is respected, since tolerances are automatically interpreted to yield tolerance zones and datum reference frames while their meaning and syntax are validated.

With this global approach to tolerance modelling, multiple kinematic chains can be coupled to analyse tolerance zones and carry out tolerance transfer, particularly for manufacturing-engineering applications, where tolerance analysis is critical for proper processplanning generation. Complete product modelling and true CAD/CAM integration are impossible without a proper tolerancing model, such as the proposed kinematic model, integrated within an exact solid modeller.

\section{REFERENCES}

1 Requicha, A A G 'Part and assembly description languages. Part I: Dimensioning and tolerancing' Technical Memo 19 Production Automation Project, University of Rochester, USA (May 1977)

2 Requicha A A G 'Toward a theory of geometric tolerancing' Technical Memo 40 Production Automation Project, University of Rochester, USA (Mar 1983)

3 Etesami, $F$ 'Tolerance verification through manufactured part modeling' J. Manuf. Syst. Vol 7 No 3 (1988) pp 223-232

4 Requicha, A A G and Chan, S C 'Representation of geometric features, tolerances, and attributes in solid modellers based on constructive geometry' IEEE J. Robot. Automat. Vol RA-2 No 3 (1986) pp 156-166

5 Kimura, F, Suzuki, $H$ and Wingard, $L$ 'A uniform approach to dimensioning and tolerancing in product modeling' in $\mathrm{Bo}, \mathrm{K}$, Estensen, L, Falster, P and Warman, E A (Eds.) Computer Applications in Production and Engineering North-Holland (1987) pp 165-178

6 Johnson, $\mathrm{R} \mathrm{H}$ (and associates) 'Dimensioning and tolerancing final report' CAM-I, USA (1985)

7 Jayaraman, $\mathrm{R}$ and Srinivasan, $\mathrm{V}$ 'Geometric tolerancing. Part I: Virtual boundary requirements' IBM J. Res. \& Develop. Vol 33 No 2 (1989) pp 90-104

8 Wirtz, A 'Vectorial tolerancing for production quality control and functional analysis in design' CIRP Int. Working Sem. Computer-Aided Tolerancing Pennsylvania State University, USA (16, 17 May 1991) pp 77-84

9 Turner, J U 'Tolerances in computer-aided geometric design' $P h D$ Thesis Rensselaer Polytechnic Institute, USA (1987)

10 Turner, J U 'A feasibility space approach for automated tolerancing' J. Eng. Indust. Vol 115 (Aug 1993) pp 341-346

11 Martino, P M Application of variational geometry to the analysis of tolerances in solid models' PhD Thesis Rensselaer Polytechnic Institute, USA (1988)

12 Roy, U and Liu, C R 'Feature-based representational scheme of a solid modeler for providing dimensioning and tolerancing information' Robot. Comput. Integr. Manuf. Vol 4 No 3/4 (1988) pp 335-345

13 Shah, J J and Miller, D W 'A structure for supporting geometric tolerances in product definition systems for CIM' Manuf. Rev. Vol 3 No 1 (1990) pp 23-31

14 Bernstein, N S and Preiss, $\mathrm{K}$ 'Representation of tolerance information in solid models' Proc. 1989 ASME 15th Design Automation Conf. ASME (1989) pp 37-48

15 Fleming, A D A representation for geometrically toleranced parts' in Woodwark, J (Ed.) Geometric Reasoning Clarendon Press, UK (1989) pp 141-167

16 Clement, A, Desrochers, A and Riviere, A 'Theory and practice of 3-D tolerancing for assembly' CIRP Int. Working Sem. Computer-Aided Tolerancing Pennsylvania State University, USA (16, 17 May 1991) pp 25-57

17 Desrochers, A 'Modèle conceptual du dimensionnement et du tolérancement des mécanismes. Représentation dans les systèmes CFAO' Thèse de Doctorat École Centrale Paris, France (1991)

18 Srinivasan, V 'A geometer grapples with tolerancing standards' CIRP Int. Working Sem. Computer-Aided Tolerancing Pennsylvania State University, USA (16, 17 May 1991) pp 191-200

19 Shepherd, D W 'Tolerancing applications for CAD: a tutorial' Fall Nat. Design Engineering. Show \& Conf. New York, USA (16-18 Sep 1986) pp 29-32

20 Pruitt, G 'Geometric dimensioning and tolerancing' Manuf. Eng. Vol 97 No 1 (1986) pp 39-45

21 Garcia, D L and St Charles, D P 'Automating GD\&T' Quality (Jun 1988) pp 56-58 
22 Guilford, $\mathrm{J}$ and Turner, $\mathrm{J}$ 'Representational primitives for geometric tolerancing' Comput.-Aided Des. Vol 25 No 9 (1993) pp 577-586

23 'Dimensioning and tolerancing' ANSI Standard Y14.5M-1982 ASME, USA (1982)

24 Krulikowski, A 'Geometric dimensioning and tolerancing: a self-study workbook' Effective Training, Westland, MI, USA (1986)

25 Hillyard, $\mathrm{R}$ 'The BUILD group of solid modelers' IEEE Comput. Graph. \& Applic. Vol 2 No 2 (1982) pp 43-52

26 Rivest, L, Fortin, $C$ and Desrochers, A 'Tolerance modeling for 3D analysis - presenting a kinematic formulation' CIRP Sem. Computer Aided Tolerancing ENS Cachan, France (27, $28 \mathrm{Apr}$ 1993) pp 51-73

27 Brown, C M 'PADL-2: a technical summary' IEEE Comput. Graph. \& Applic. Vol 2 No 2 (1982) pp 69-84

28 'ACIS interface guide' Spatial Technology, Boulder, CO, USA (1992)

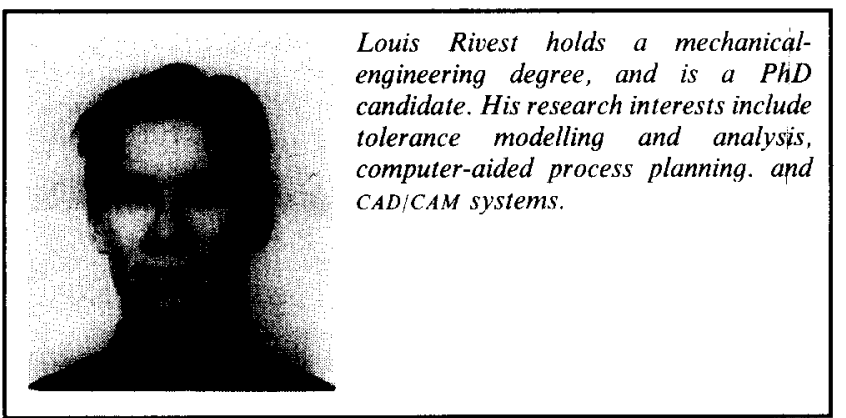

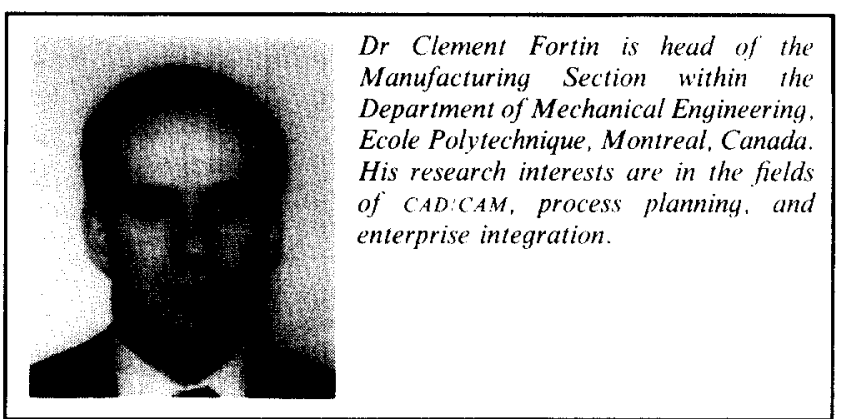

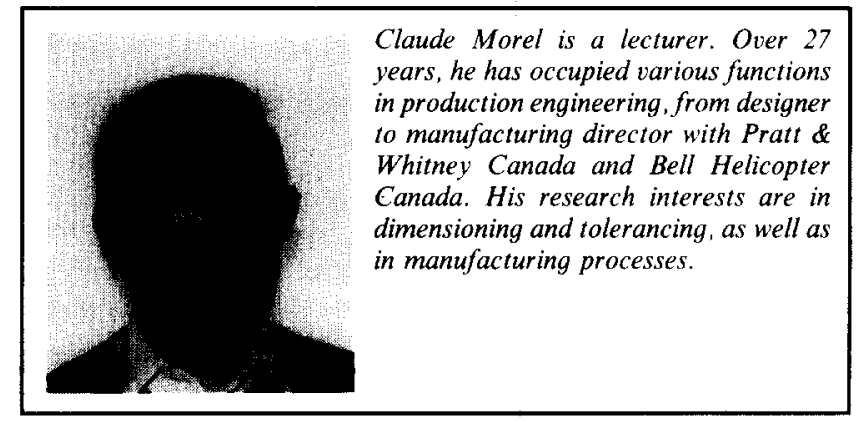

